\title{
The Template Synthesis of Double Coaxial Carbon Nanotubes with Nitrogen-Doped and Boron-Doped Multiwalls
}

\author{
Quanhong Yang, Weihua Xu, Akira Tomita, and Takashi Kyotani* \\ Institute of Multidisciplinary Research for advanced Materials, Tohoku University, Sendai \\ 980-8577, Japan
}

\section{Supporting information}

Electrical resistance measurement. The electrical resistance of the carbon-coated AAO films (CNT/AAO composites) was measured by a two-terminal method as shown in Figure S1. All the nanotubes prepared by the template technique were embedded in the nanochannels of AAO templates and parallel to each other as a result. We performed oxygen plasma treatment $(100 \mathrm{~W}, 2 \mathrm{~min})$ for CNT/AAO to remove the carbon layers deposited on the outer surface of AAO template. We coated both sides of a plasma-treated CNT/AAO composite film with silver paste (Dotite 550, Fujikura Kasei), to which two silver wires are attached. A potential (-1.0 to $1.0 \mathrm{~V})$ is applied to both surfaces of the film through the two conducting wires and the I-V characteristics were measured at a temperature of $25^{\circ} \mathrm{C}$. According to the I-V curves, the resistances were obtained and then the specific resistivity of the prepared CNTs was induced. For each case, five samples were measured to obtain the average resistance $\left(R_{m}\right)$, after the error correction by subtracting the contact resistance of conducting wires and silver paste). The area (denoted as A) of each side of CNT-AAO film employed is about $0.5 \mathrm{~cm}^{2}$, and the number ( $\mathrm{n}$ ) of nanotubes in an AAO template per unit area is estimated as $5 \times 10^{10} / \mathrm{cm}^{2}$ according to the SEM observation. Apparently, the resistance $(\mathrm{R})$ of an individual nanotube is determined from $\mathrm{Rm}, \mathrm{n}$ and $\mathrm{A}$ by the equation: $\mathrm{R}=\mathrm{nAR}$. Then the specific resistivity $(\rho)$ of $\mathrm{CNT}$ is calculated according to the equation: $\rho=\mathrm{RS} / \mathrm{L}$ ( $\mathrm{S}, \mathrm{L}$ : cross sectional area and length of an individual CNT, respectively).

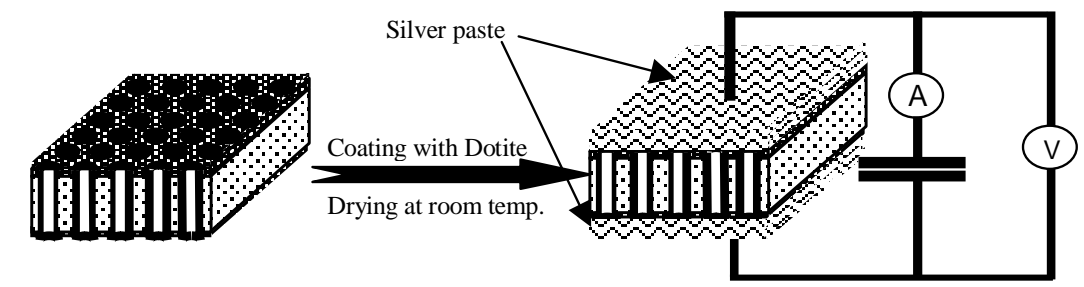

CNT/AAO (carbon coated AAO)

CNT-AAO with both sides of silver paste

Figure S1. Schematic diagram of electrical conductance measurement of template-synthesized CNTs 